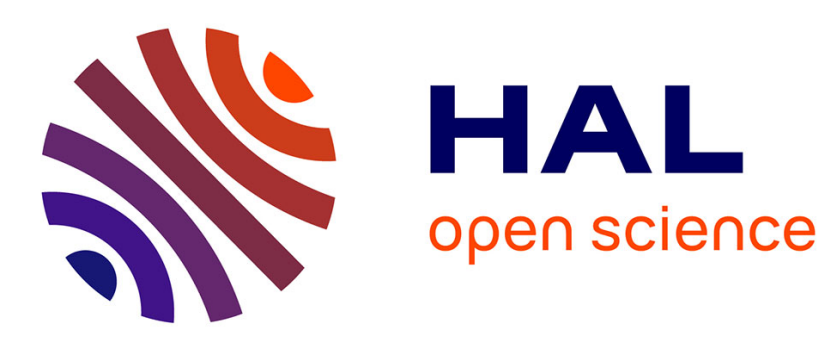

\title{
Rassembleur à harmonique 3 simulé
}

C. Goldstein, A. Laisné

\section{- To cite this version:}

C. Goldstein, A. Laisné. Rassembleur à harmonique 3 simulé. Revue de Physique Appliquée, 1969, 4

(2), pp.183-184. 10.1051/rphysap:0196900402018300 . jpa-00243208

\section{HAL Id: jpa-00243208 https://hal.science/jpa-00243208}

Submitted on 1 Jan 1969

HAL is a multi-disciplinary open access archive for the deposit and dissemination of scientific research documents, whether they are published or not. The documents may come from teaching and research institutions in France or abroad, or from public or private research centers.
L'archive ouverte pluridisciplinaire HAL, est destinée au dépôt et à la diffusion de documents scientifiques de niveau recherche, publiés ou non, émanant des établissements d'enseignement et de recherche français ou étrangers, des laboratoires publics ou privés. 


\title{
RASSEMBLEUR A HARMONIQUE 3 SIMULÉ
}

\author{
G. GOLDSTEIN et A. LAISNÉ, \\ Institut de Physique Nucléaire, 91-Orsay.
}

\begin{abstract}
Résumé. - Nous proposons un rassembleur à haute efficacité utilisant le fondamental et l'harmonique 2 pour simuler l'harmonique 3 . Le rendement théorique d'un tel système est $\eta=88 \%$ au lieu de $65 \%$ avec un rassembleur classique. Pratiquement, on peut espérer un facteur d'amélioration compris entre 4 et 5 .
\end{abstract}

Abstract. - We propose a high efficiency buncher using the fundamental frequency and its second harmonic to simulate the third one. The theoretical efficiency of this system is $88 \%$ instead of $65 \%$ with the classical one. Practically, it is possible to obtain a gain between 4 and 5 instead of 3 with the classical buncher.

Le but du rassembleur est d'introduire le maximum de particules à l'intérieur du diagramme d'acceptance de phase de l'accélérateur linéaire $\left(\varphi_{\mathrm{s}}=-20^{\circ}, \mathrm{fig} .1\right)$. Sans rassembleur, le rendement d'acceptance est

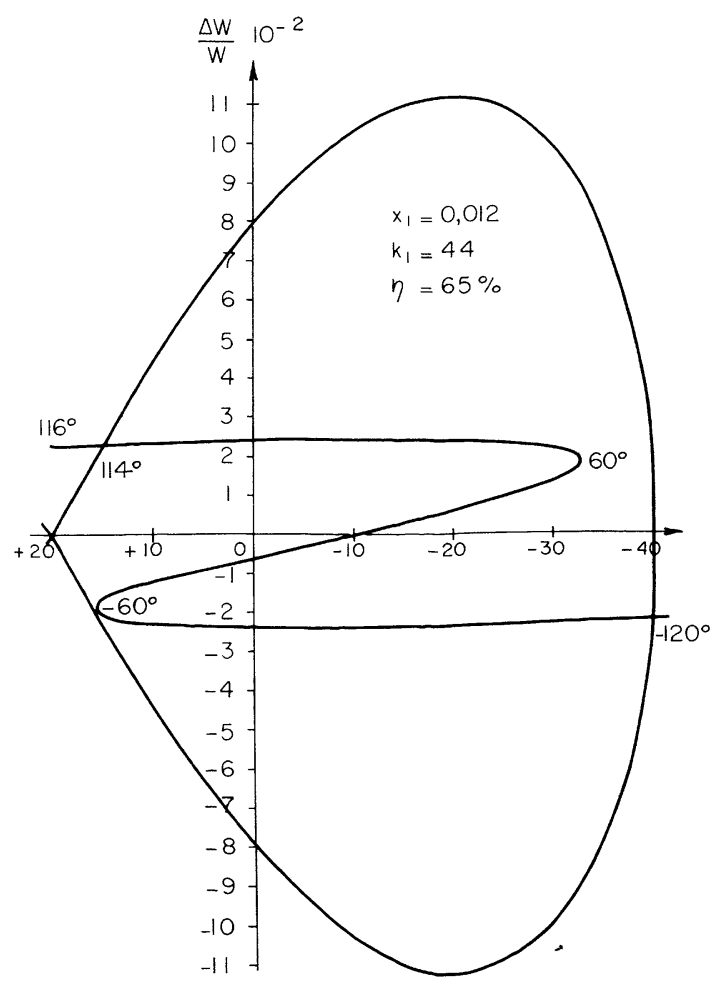

FIG. 1. $\eta=\frac{3\left|\varphi_{\mathrm{s}}\right|}{2 \pi}=16,6 \%$. Il est facile de montrer [1] qu'en l'absence de charge d'espace le rassembleur donnant un regroupement total en phase doit communiquer à un ion quelconque un accroissement algébrique de vitesse proportionnel à son déphasage par rapport à l'ion synchrone, l'ion synchrone étant défini comme étant celui qui ne prend aucune modulation de vitesse dans le système et arrive dans l'accélérateur à la phase de synchronisme. On montre que la tension de modulation à réaliser est la dent de scie, ce qui est difficile.

I. Rassembleur classique. - L'approximation la plus classique de la dent de scie est la sinusoïde à la fréquence de l'accélérateur. Si l'on appelle $V_{1}$ la tension sur l'électrode du rassembleur, $V_{0}$ la tension d'injection, $x_{1}=\frac{V_{1}}{V_{0}}$ et si la longueur de glissement est $L_{1}=\frac{K_{1}}{2 f} \sqrt{\frac{2 q V_{0}}{m}}$, on peut montrer que le regroupement maximal est tel que $\eta=65 \%$ (fig. 1). On admet généralement que le gain est d'un facteur 3 .

II. Rassembleur à harmonique 2 [2]. - Si l'on place près du rassembleur classique une seconde électrode à la fréquence double dont la tension appliquée $V_{2}$ $\left(x_{2}=\frac{V_{2}}{V_{0}}\right)$ a une amplitude moitié de celle du fondamental, on obtient la courbe de la figure 2 , où $K_{1}$ et $K_{2}$ sont des coefficients proportionnels respectivement 


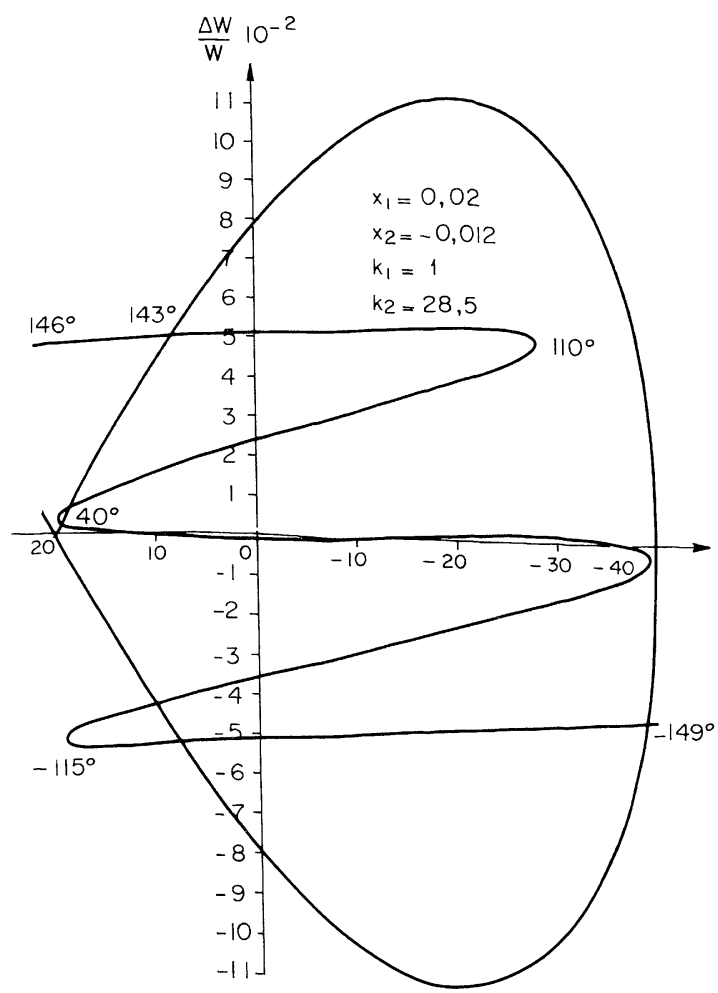

FIG. 2.

à la distance entre les deux électrodes accélératrices et à l'espace de glissement. Le rendement théorique s'est accru : $\eta=81 \%$.

III. Rassembleur à harmonique 3 simulé [3]. Si maintenant on fait croître $K_{1}$, on voit (fig. 3) que l'on passe par un optimum $\eta=88 \%$. La décomposition en fonction de Bessel de la dispersion en énergie introduite par un tel rassembleur montre que l'on a simulé un harmonique 3 , d'amplitude voisine de celle du troisième harmonique de la décomposition en série de Fourier de la dent de scie, c'est-à-dire que l'on a maintenant les trois premiers termes de la décomposition avec les bonnes phases (l'harmonique 4 existe avec le bon signe mais n'a que la valeur moitié de la valeur nécessaire, l'harmonique 5 n'a que le $1 / 7 \ldots)$.

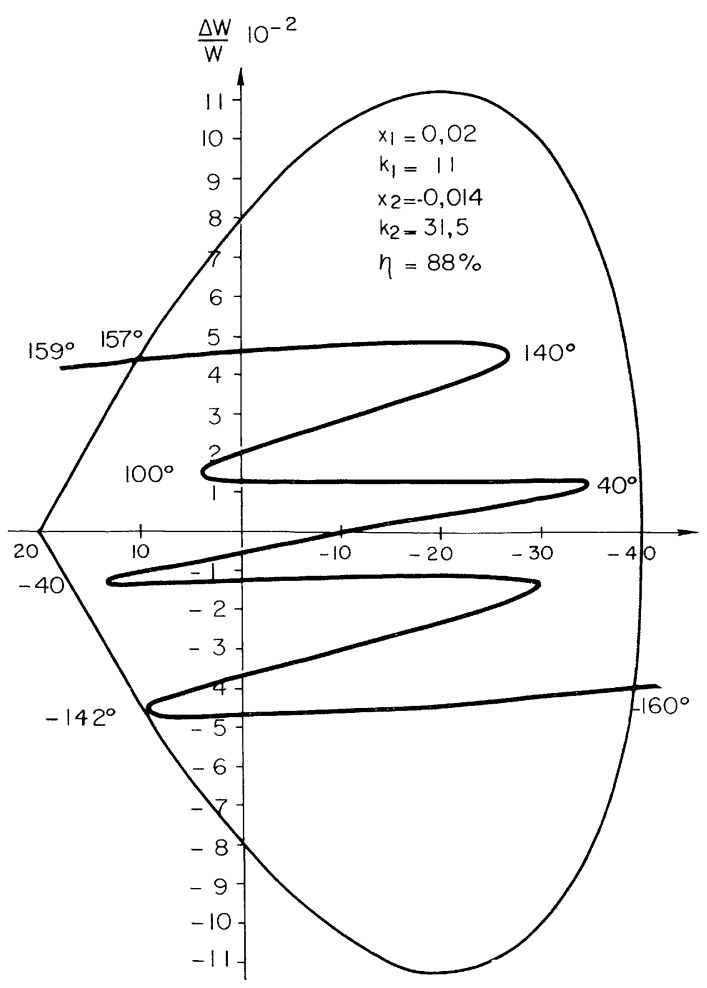

FIG. 3.

Ce rassembleur a été essayé sur une maquette d'accélérateur de 10 électrodes. Le rassembleur classique ( fig. 1) a donné un gain de 3 , le rassembleur à harmonique 3 simulé, un gain de 5 ; il est raisonnable d'espérer sur l'accélérateur linéaire à ions lourds d'Orsay un gain compris entre 4 et 5 .

\section{BIBLIOGRAPHIE}

[1] GoldDSTEIn (C.) et LaIsné (A.), Étude du rassembleur de l'accélérateur linéaire à ions lourds d'Orsay, Rapport interne (févr. 1967).

[2] Myers (W.), The ANL Second Harmonic Buncher, Proc. 1966 Linear Accelerator Conf.

[3] Golddstein (C.) et LaISnÉ (A.), Nucl. Instr. Methods, $1968,61,221$. 